\title{
Modeling and performance evaluations of Alamouti technique in a single frequency network for DVB-T2
}

\author{
Mokhtar Tormos ${ }^{1,2}$, Camel Tanougast ${ }^{2 *}$, Abbas Dandache $^{2}$, Denis Masse ${ }^{1}$ and Pierre Kasser ${ }^{1}$
}

\begin{abstract}
Alamouti space-frequency coding provides additional frequency diversity, especially in multipath propagation environments. This article addresses a new modeling of the Alamouti code multiple input single output (MISO) in a single frequency network(SFN) for two, three, and four transmitters, and different types of coding and modulations in the DVB-T2 (Digital Video Broadcasting Terrestrial second generation). Classical SFN, Alamouti MISO, and combined SFN with Alamouti MISO (called SFN-2x1 Alamouti MISO) are compared and analyzed. Performance evaluations are made for two, three, or four transmission antennas through 0-dB Echo profile, TU6 mobile channel, unbalanced received power and rotated constellation. The obtained results show clearly that the performance of an SFN network using 0-dB Echo profile with Alamouti MISO is only better than a pure SFN for two and three antennas. Moreover, an SFN based on four antennas (receiving from four antennas) has almost the same performance as SFN-2x1 Alamouti MISO.
\end{abstract}

Keywords: DVB-T2, 2x1 Alamouti MISO, Single frequency network (SFN), Path gain, 0-dB echo channel

\section{Introduction}

Although the Digital Video Broadcasting Terrestrial standard (DVB-T) supports singlefrequency networks (SFNs) to get better reception quality [1-3], the presence of similar-strength signals from two transmitters in a network causes a significant loss of margin because the resulting channel can have deep "notches". DVB-T and DVB-T2 (Digital Video Broadcasting Terrestrial second generation) define an SFN corresponding to 0-dB Echo profile [4]. DVB-T2 incorporates the Alamoutispace-frequency [5-9] coded orthogonal frequency division multiplexing systems (COFDM) with a pair of transmitters [10] to eliminate these notches and has better performance. The Alamouti coding is a Multiple Input Single Output (two transmit antennas and a single receive antenna-2x1 MISO) system, in which every constellation point is transmitted by transmitters [5]. The first transmitter transmits the constellations without modifications while the second one transmits a slightly modified version of each pair of constellations in the reverse order in frequency.

\footnotetext{
* Correspondence: Camel.Tanougast@univ-lorraine.fr

${ }^{2}$ Télédiffusion de France (TDF), 1 rue Marconi, 57070, Metz, France Full list of author information is available at the end of the article
}

Previous works have given the performance of DVB-T2 in an SFN network, F1 Ricean channel, P1 Rayleigh channel [4] and the performance of MISO technique [11]. Others' works have considered the mobility in $D V B-H$ and $T-D M B$ [12], the performance of TU6 for DVB-T2 for Doppler 80 $\mathrm{Hz}$ and present an envisaged result of TU6 for low Doppler $10 \mathrm{~Hz}$ for DVB-T2 [13,14]. The performance of MISO in TU6 for CD3 [15] receiver is also given in [15]. A number of studies have discussed the performance evaluations and shown the benefit of Alamouti MISO transmission [11]. Actually, there are not any performance evaluations of SFN-MISO for $0 \mathrm{~dB}$ echo and TU6 channel in DVB-T2. The study in this article is targeted at this goal. More precisely, this article gives performance evaluations and comparison between a classical SFN network and a distributed SFN-MISO network depending on the number of transmitters using different coding rate and QAM modulation for a DVB-T2 system and different reception condition (fixed and mobile). Our study and evaluation results seek to determine the transmission topologies, for which the usage of the Alamouti technique in an SFN network of different number of transmitters is efficient. Indeed, these results are very important for the broadcaster to be used for optimal 
network planning and management. Our obtained simulation results show the value of using the Alamouti technique in an SFN network based on two or three antennas in DVB-T2.

The remainder of this article is organized as follows. Section 2 gives a new model of an SFN-2x1 Alamouti MISO transmission for two, three, and four transmitters. Section 3 includes modeling and simulation results with a detailed analysis of performance of classical mobile channel (TU6) for Doppler frequency of $80 \mathrm{~Hz}$ in a classical SFN network on TU6 channel (SFN-TU6) compared to an SFN2x1 Alamouti MISO on TU6 channel (SFN-MISO-TU6) for DVB-T2. Sections 4 and 5 evaluate the signal-to-noise ratio (SNR) performance of the unbalanced received power and rotated constellation effects on the SFN and SFN-2x1 Alamouti MISO networks, respectively. Among all configurations used for the different studied cases, we analyze the different reception zones required for the simulation using the CSP DVB-T2 platform, for which evaluation results are shown. Finally, conclusion and future works are given in Section 6.

\section{Proposed modeling of the Alamouti MISO in DVB-T2}

We study the performance of SFN-2x1 Alamouti MISO compared to classical SFN for two, three, and four transmitters. We consider a new DVB-T2 configuration with the same SNR as fixed reception of the DVB-T configuration currently used for $H D$ multiplex in France [2].

\subsection{Transmission model}

We propose a new transmission model based on two, three, and four distributed antennas, which combine the actual SFN network with Alamouti MISO diversity $[11,16]$. The proposed modeling is based on two, three, and four transmitters where each transmitter has two types of transmission. The first one is denoted mode " $\mathrm{A}$ " where the symbols transmitted are $a_{m, 1, p}$ and $a_{m, 1, p+1}$ at two adjacent orthogonal frequency division multiplexing (OFDM) carriers $k$ and $k+1$, respectively. The second transmission type is denoted mode " $\mathrm{B}$ " where the conjugate symbols $-a^{*}{ }_{m, l, p+1}$ and $a^{*}{ }_{m, l, p}$ at the same carriers $k$ and $k+1$, respectively. These symbols are transmitted as defined by Alamouti MISO based on two transmit antennas and a single receive antenna (2x1 Alamouti scheme) $[11,17]$. As specified by the standard DVB-T2 [4], indices $m, l$, and $p$ are the T2-frame number, the symbol within the frame and the data cell within the symbol prior to frequency interleaving and pilot insertion, respectively. This proposed model allows the analysis of three transmission cases. The first one is the SFN (normal transmission) where transmitters transmit in mode "A". The second is the 2x1 Alamouti MISO (diversity transmission) where one transmitter transmits in the mode "A" while the second transmits in the mode "B". The third case is the SFN-2x1 Alamouti MISO based on three or four antennas where transmitters can transmit either in the mode "A" or "B". This case is a combined transmission where we find one group of antennas which transmits the mode " $A$ " and a second one which transmits the mode "B". Each group forms an SFN network while the combination of these two groups forms a diversity transmission. Figure 1 illustrates our modeling approach. The different reception zones depending on the transmission cases are detailed in this figure. Therefore, the reception areas from $1,2,3$, and 4 antennas for these different reception zones are given.

The reception zones shown in Figure 1 are detailed as follows.

1) The zones labeled (1) is the reception zone from one antenna (transmission mode "A" or mode "B") allowing normal transmissions.

2) The reception zone from two antennas and divided in two sub-cases allowing diversity transmissions:

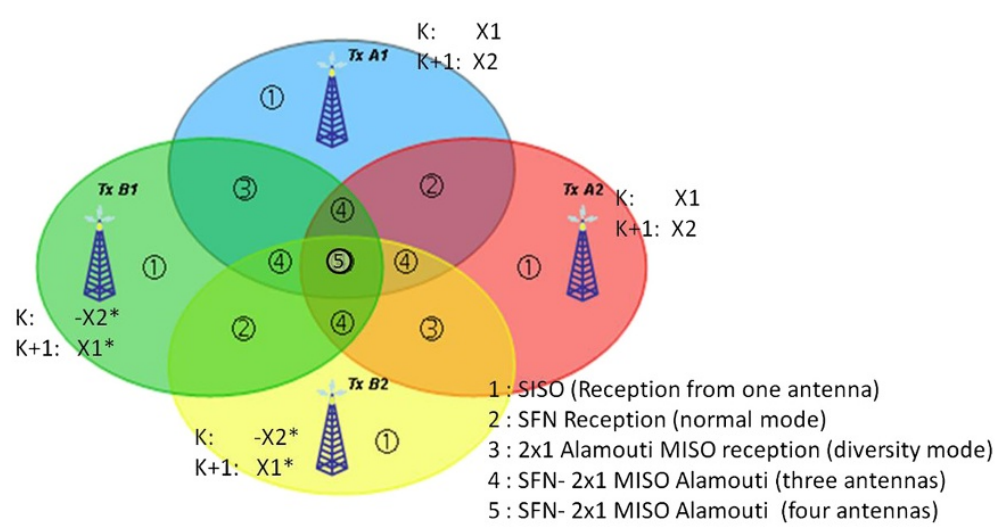

Figure 1 Modeling of different proposed transmission cases. 
i). The zones labeled (2) are the reception zones based on the $2 \times 1$ Alamouti MISO from two antennas $\left(A_{1}+B_{1}\right.$ or $\left.A_{2}+B_{2}\right)$.

ii). The zones labeled (3) correspond to reception zones based on an SFN network from two pair of antennas $\left(A_{1}+A_{2}\right.$ or $\left.B_{1}+B_{2}\right)$ and where each pair of antennas $\left(A_{1}+A_{2}\right)$ and $\left(B_{1}+B_{2}\right)$ refer to the mode " $A$ " and " $B$ " transmissions, respectively.

3) The zones labeled (4) refer to the reception zones from three antennas $\left(A_{1}+A_{2}+B_{1}\right.$ or $\left.A_{1}+B_{1}+B_{2}\right)$ that represent the 2x1 Alamouti MISO in an SFN. These zones are considered as combined SFN-2x1 Alamouti MISO networks allowing diversity transmissions in an SFN network and according with the following configuration cases:

i). The antenna group $\left(A_{1}+A_{2}\right)$ is considered as one antenna $A_{12}$ that corresponds to an SFN network in the mode " $A$ " transmission while the antenna $B_{1}$ is in the mode " $B$ " transmission. It results in a diversity transmission.

ii). Reciprocally, the antenna $A_{1}$ is in the mode " $A$ " transmission while the antenna group $\left(B_{1}+B_{2}\right)$ is considered as single antenna $B_{12}$ that corresponds to an SFN network. Similarly, this combined network allows a diversity transmission.

4) The zone (5) is the reception zone from four antennas $\left(A_{1}+A_{2}+B_{1}+B_{2}\right)$, which represent two combined SFN-2x1 Alamouti MISO allowing diversity transmission from the considered equivalent pair of antennas $\left(A_{12}+B_{12}\right)$.

Three cases are studied in this work. In the first case, we have analyzed the reception signal from two antennas $A_{1}+B_{1}$. In the second case, the reception signals from three antennas $A_{1}+A_{2}+B_{1}$ or $A_{1}+B_{1}+B_{2}$ were analyzed. Finally, the reception from four antennas $A_{1}+A_{2}+B_{1}+B_{2}$ were studied in the third case.

\subsection{Model equations}

To reconstruct the signal at each point in the reception zone in the case of $2 \times 1$ Alamouti MISO (diversity transmission), we formulate the reception for two transmitters by a single antenna at two different carriers $k$ and $k+1$. The reconstruction of the transmitted signal is achieved from two received signals $y_{1}$ and $y_{2}$ expressed by the vector $\boldsymbol{Y}$ and specified by Equations (1) and (2), at the carriers $k$ and $k+1$, respectively:

$$
\begin{aligned}
y_{1}= & h_{1(m, l, p)} \cdot a_{(m, l, p)}-h_{2(m, l, p)} \cdot a^{*}{ }_{(m, l, p+1)}+n_{1} / 2 \\
y_{2}= & h_{1(m, l, p+1)} \cdot a_{(m, l, p+1)}+h_{2(m, l, p+1)} \cdot a^{*}{ }_{(m, l, p)} \\
& +n_{1} / 2
\end{aligned}
$$

where $y_{1}$ and $y_{2}$ are the received signals at the sub-carriers $k$ and $k+1 . h_{1}$ and $h_{2}$ are the coefficients of the channel defined by the matrix $\mathbf{H}$ between the two transmit antennas $A_{1}$ and $B_{1}$ and the receiver. The component $n_{1}$ is the complex random variable and represents the receiver noise and interference defined by the vector $\boldsymbol{n}$. We can represent these equations in the following matrix form:

$$
y=\mathbf{H} \cdot \mathbf{a}+\mathbf{n}
$$

where $\mathbf{y}=\left(\begin{array}{c}y_{1} \\ y^{*}{ }_{2}\end{array}\right), \mathbf{H}=\left(\begin{array}{cc}h_{1(m, l, p)} & -h_{2(m, l, p)} \\ h_{2}{ }^{*}(m, l, p+1) & h_{1}{ }^{*}{ }_{(m, l, p+1)}\end{array}\right), \mathbf{n}=$ $\left(\begin{array}{c}n_{1} / 2 \\ n^{*}{ }_{1} / 2\end{array}\right)$, and $\mathbf{a}=\left(\begin{array}{c}a_{(m, l, p)} \\ a^{*}{ }_{(m, l, p+1)}\end{array}\right)$, is the data transmission vector.

For an SFN transmission model, more precisely $0 \mathrm{~dB}$ Echo, the received signal $\mathrm{y}(t)$ is computed as follows:

$$
y(t)=h_{1} \cdot e(t)+h_{2} \cdot e(t-\tau)
$$

where $h_{1}$ and $h_{2}$ are the channel coefficients between the signal $e(t), e(t-\tau)$, and the receiver. In the frequency domain, the received signal is given by the following expression:

$$
y(f)=\left|h_{1(m, l, p)}\right| \cdot E(f)+\left|h_{3(m, l, p)}\right| \cdot e(f) \cdot e^{-j 2 \pi f \tau}
$$

We deduce the received signals at the sub-carriers $k$ and $k+1$ as follows:

$$
\begin{aligned}
y_{1} & =\left(h_{1(m, l, p)}+h_{3(m, l, p)}\right) \cdot a_{(m, l, p)} \\
& =\left(\mid h_{1\left(m, j, p|+| h_{3(m, l, p)} \mid e^{-j 2 \pi f \tau}\right) \cdot a_{(m, l, p)}}(6)\right. \\
y_{2} & =\left(h_{2(m, l, p)}+h_{4(m, l, p)}\right) \cdot a^{*}{ }_{(m, l, p+1)} \\
& =\left(\left|h_{2(m, j, p+1)}\right|+\left|h_{4(m, l, p+1)}\right| e^{-j 2 \pi f \tau}\right) \cdot a^{*}{ }_{(m, l, p+1)}
\end{aligned}
$$

We can represent these equations in the matrix form as defined by Equation (3) and where the matrix channel is given as follows:

$$
H=\left(\begin{array}{cc}
\left(\left|h_{1(m, j, p|+|}\right| h_{3(m, l, p)} \mid \cdot e^{-j 2 \pi f \tau_{\tau}}\right) & 0 \\
0 & \left.\left|h_{2(m, j, p+1)}\right|+\left|h_{4(m, l, p+1)}\right| \cdot e^{-j 2 \pi f \tau}\right)
\end{array}\right)
$$

Our new proposal model is based on the superposition of an SFN with 2x1 Alamouti MISO in the case of four transmitters as depicted in Figure 2. As shown in this figure, the signals received from transmitters $A_{1} / A_{2}$ and $B_{1} / B_{2}$ are treated as pair of SFN combined in diversity transmission.

Therefore, applying Equations (1), (2), (6), and (7) in the case of a reception zone based on four antennas 


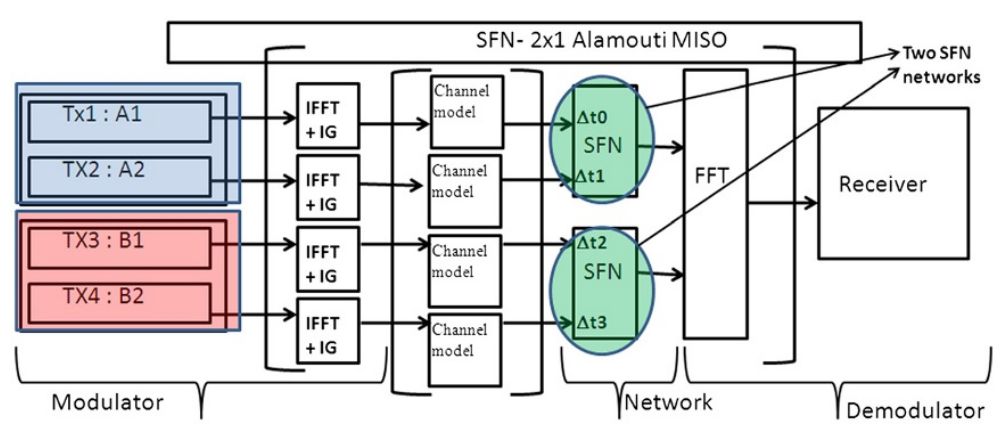

Figure 2 Proposed SFN-2x1 Alamouti MISO transmission model based on four transmitters.

$A_{1}+A_{2}+B_{1}+B_{2}$, we formulate the SFN-2x1 Alamouti MISO model by Equations (9) and (10), which are obtained at carriers $k$ and $k+1$, respectively

$$
\begin{aligned}
y_{1}= & \left(h_{1(m, l, p)}+h_{3(m, l, p)}\right) \cdot a_{(m, l, p)} \\
& -\left(h_{2(m, l, p)}+h_{4(m, l, p)}\right) \cdot a^{*}{ }_{m, l, p+1}+n_{1} / 2 \\
y_{2}= & \left(h_{2(m, l, p+1)}+h_{4(m, l, p+1)}\right) \cdot a^{*}{ }_{(m, l, p)} \\
& +\left(h_{1(m, l, p+1)}+h_{3(m, l, p+1)}\right) \cdot a_{m, l, p+1} \\
& +h_{1} / 2
\end{aligned}
$$

where $h_{3}$ and $h_{4}$ are the coefficients of the channel between the two other transmitters $A_{2}$ and $B_{2}$ and the receiver. For simplification, we define $h_{1}{ }^{\prime}$ and $h_{2}{ }^{\prime}$ as follows:

$$
\begin{aligned}
& h_{1}^{\prime}=h_{1}+h_{3} \\
& h_{2}^{\prime}=h_{2}+h_{4}
\end{aligned}
$$

Note that in the case of three antennas $h_{2}$ 'is equal to $h_{2}$. Thus, the matrix $\mathbf{H}$ and Equations (9) and (10) at carriers $k$ and $k+1$ can be written as follows:

$$
\begin{aligned}
\mathbf{H}= & \left(\begin{array}{cc}
h_{1}^{\prime}{ }_{(m, l, p)} & -h_{2}^{\prime}{ }_{(m, l, p)} \\
h_{2}{ }^{*}{ }_{(m, l, p+1)} & h_{1}{ }^{*}{ }_{(m, l, p+1)}
\end{array}\right) \\
y_{1}= & h_{1(m, l, p)}^{\prime} \cdot a_{(m, l, p)}-h_{2(m, l, p)}^{\prime} \cdot a^{*}{ }_{m, l, p+1}+n_{1} / 2 \\
y_{2}= & h_{2(m, l, p+1)}^{\prime} \cdot a^{*}{ }_{(m, l, p)}+h_{1(m, l, p+1)}^{\prime} \cdot a_{m, l, p+1} \\
& +n_{1} / 2
\end{aligned}
$$

The DVB-T2 receiver makes measurements of the channel using pilots and then by interpolating between these measurements the estimates of the channel response for every OFDM cell [4] are constructed. After estimating the channel, a Zero-Forcing equalizer for the signal model in Equation (3) provides the unbiased estimates $m, l, p, m, l, p+1$ [18] and is given by

$$
\hat{\mathrm{A}}=\left(\begin{array}{c}
m, l, p \\
*{ }_{m, l, p+1}
\end{array}\right)=\left(H^{*} \cdot H\right)^{-1} H^{*} \cdot \mathbf{y}
$$

Note that the first term $\left(\mathrm{H}^{* \prime} \cdot \mathrm{H}^{-1}\right)$ in Equation (16) corresponds to the equalization part whereas the term $\mathrm{H}^{*}$ is the actual matched filter receiver for the vector signal model defined in Equation (3) [7]. By using the structure of the channel matrix in $\mathbf{H}$ the entire matched filter matrix can be written as follows:

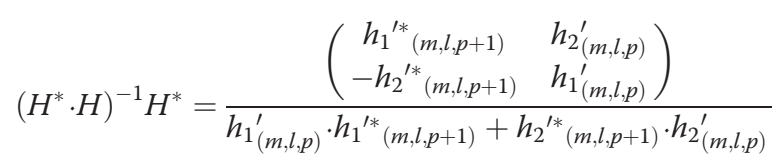

Consequently, the estimates $\hat{A}$ for the transmitted signal can be computed as follows:

$$
\hat{a}_{m, l, p}=\frac{h_{1}{ }^{*}{ }_{(m, l, p+1)} \cdot y_{1}+h_{2}^{\prime}{ }_{(m, l, p)} \cdot y^{*}{ }_{2}}{h_{{ }_{(m, l, p)}}^{\prime} \cdot h_{1}{ }^{*}{ }_{(m, l, p+1)}+h_{2}{ }^{*}{ }_{(m, l, p+1)} h_{2}^{\prime}{ }_{(m, l, p)}}
$$

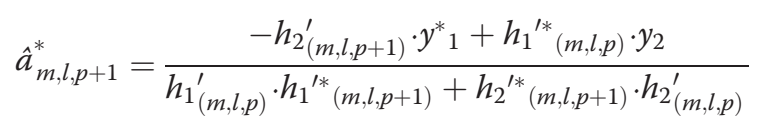

Note that for the flat fading case where $h_{1}{ }^{\prime}{ }_{(m, l, p)}=h_{1}{ }^{\prime}$ ${ }^{(m, l, p+1)}$ and $h_{2}{ }^{\prime}{ }_{(m, l, p)}=h_{2}{ }^{\prime}{ }(m, l, p+1)$, the resulting channel matrix $\mathbf{H}$ is orthogonal $[7,17]$. More precisely, the transmission model assumes that the channel does not vary during the two consecutive OFDM Symbols. Therefore, the resulting channel matrix $\mathbf{H}$ is orthogonal as explained by the following equation:

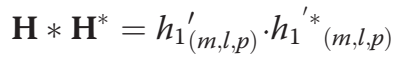

$$
\begin{aligned}
& +h_{2}{ }^{*}{ }_{(m, l, p)} \cdot h_{2}{ }_{(m, l, p)}\left(\begin{array}{ll}
1 & 0 \\
0 & 1
\end{array}\right) \\
& =\left(\left|h_{1}{ }^{\prime}(m, l, p)\right|^{2}+\left|h_{2}{ }^{\prime}(m, l, p)\right|^{2}\right) \cdot \mathbf{I} \text {, }
\end{aligned}
$$

where the determinant of the Matrix $\mathbf{H}^{*} \mathbf{H}^{*}$ is null (det $\left.\left(\mathbf{H}^{*} \mathbf{H}^{*}\right)=0\right)$. I represents the identity Matrix. 
For the frequency selective case where $\left(h_{1}{ }^{\prime}{ }_{(m, l, p)} \neq h_{1}{ }^{\prime}\right.$ ${ }^{(m, l, p+1)}$ and $\left.h_{2}{ }^{\prime}{ }_{(m, l, p)} \neq h_{2}{ }^{\prime}{ }^{\prime}(m, l, p+1)\right)$, the determinant of the Matrix $\mathbf{H}^{*} \mathbf{H}^{*}$ is not null $\left(\operatorname{det}\left(\mathbf{H}^{*} \cdot \mathbf{H}\right)=0\right)$. Consequently, the channel matrix $\mathbf{H}$ is no longer orthogonal and we deduce that the matched filter receiver no longer provides the optimum solution. Therefore, the above matched filter receiver converges towards the two following expressions solutions:

$$
\begin{aligned}
& \hat{a}_{m, l, p}=\frac{h_{1}{ }^{*}{ }_{(m, l, p+1)} \cdot y_{1}+h_{2}^{\prime}{ }_{(m, l, p)} \cdot y^{*}{ }_{2}}{h_{1}^{\prime 2}{ }_{(m, l, p)}+h_{2}{ }^{2 *}{ }_{(m, l, p+1)}}
\end{aligned}
$$

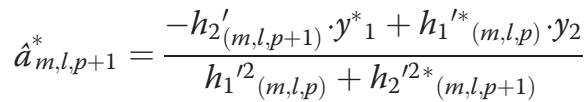

\subsection{Simulation and results}

We have compared the performance of transmission in the case of SFN, 2x1 Alamouti MISO, and SFN-2x1Alamouti MISO (see Figure 1) for different types of modulation and code rate. The Alamouti 2x1 was chosen for the DVB-T2 standard because of its rate equals 1 (performance diversity gain without losing the throughput of transmission data). Indeed, the choice of another MISO type like $3 \mathrm{x} 1$ or $4 \mathrm{x} 1$ Tarokh can lead to lose some throughput of transmission data even if it has better diversity gain performance. More precisely, orthogonal codes based on three and four transmit antennas were proposed by Tarokh et al. [19]. The 3x1 and 4x1 Tarokh codes, when implemented as distributed orthogonal space-frequency block codes for diversity gain, have lower rates than for Alamouti [19-22], which leads to a loss in throughput for transmission. In addition, the main reason to use a $2 \times 1$ Alamouti rather than a $2 \times 2$ Alamouti is to avoid additional antennas at the receiver side as clearly specified in the new DVB-NGH standard [23].

The transmission model is defined for two, three, and four distributed antennas having the same received level power for different QAM modulation. The simulation of the DVB-T2 proposed model is achieved on the CSP DVB-T2 platform [24] based on the Matlab software tool [25]. This platform validates the correct operation of the system starting from a digital stream at the DVB-T2 transmitter side and ending after its reconstruction at the receiver side passes through different types of channel (0-dB Echo, Rayleigh channel, Ricean channel).

We have considered the distributed scenario of the type 1 for the Alamouti MISO parameters with the following delay values $(\tau i)$ of $0,0.18 \Delta, 0.7 \Delta$, and $0.9 \Delta$. The parameter $\Delta$ represents the guard interval fraction. These values

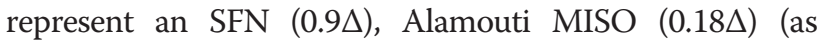
defined in [4]), and two intermediate values in the case of three and four antennas. In this case study, we suppose
Table 1 Configuration parameters for the proposed DVB-T2 modeling and simulation

\begin{tabular}{ll}
\hline Channel $8 \mathrm{MHz}$ (7.61 MHz) & ON \\
\hline Channel $8 \mathrm{MHz}(7.8 \mathrm{MHz})$ & OFF \\
Mode & $32 \mathrm{~K}$ \\
Gard interval & $1 / 32$ \\
Scattered pilot pattern & PP4 \\
LDPC Size & 64,800 \\
T2 frame length & 60 symbols \\
number of T2 frame per superframe & 2 \\
Rotated Constellations & ON \\
Code rate & $2 / 3$ \\
PAPR & OFF \\
\hline
\end{tabular}

that there is no frequency offset. The parameters that are used in this simulation are shown in Table 1.

For this configuration, the bit rate values for SISO and Alamouti MISOfor256 QAM are 36.7722875 and 36.5777251 Mbits/s, respectively. The bit rate deviation (194.5624 kbits/s) corresponding to a carrier-to-noise ratio $(C / N)$ deviation of $0.1 \mathrm{~dB}$ (by considering the Shannon capacity limit) does not cause a deviation in reception quality.

We model the reception from three antennas as follows: two antennas $A_{1}$ and $A_{2}$ model an SFN transmission. After the addition of antenna $B_{1}$ or $B_{2}$, the system constitutes an SFN-2x1 Alamouti MISO transmission (diversity transmission). The specific parameters simulation channel used are:

-For the antennas $A_{1}$ and $A_{2}$ the echo values ( $\tau_{\mathrm{I}}$ in $\left.\mu \mathrm{s}\right)$ are 0 and $0.9 \Delta$, respectively, while the third antenna $B_{1}$ or $B_{2}$ is equal to $0.18 \Delta$. We consider no frequency offset $\left(\Delta f_{i}=0 \mathrm{~Hz}\right)$.

We model the reception based on four antennas from two pairs of antennas $\left(A_{1}, A_{2}\right)$ and $\left(B_{1}, B_{2}\right)$ representing an SFN network each one. The association of these pairs of antennas models a diversity transmission.

The parameter simulation of channel values of the mode transmission for this modeling is defined as follows.

-For the transmitters $A_{1}$ and $A_{2}$ the echo values ( $\tau_{I}$ in $\mu$ s) are 0 and $0.9 \Delta$, respectively, while the antennas $B_{1}$ and $B_{2}$ are equal to $0.18 \Delta$ and $0.7 \Delta$, respectively. We consider no frequency offset $\left(\Delta f_{i}=0 \mathrm{~Hz}\right)$.

It is important to study the channel frequency response in order to see the fading due to multipath for the delay values considered. The study of the channel characteristics for 2x1 Alamouti MISO, SFN, and SFN2x1 Alamouti MISO transmissions are detailed as follows. The SFN is modeled as a direct signal $e(t)$ and its signal echo $e(t-\tau)$. From Equations (4) and (5), the 
channel path gain can be expressed by the following equation:

$$
\left|\frac{R(f)}{E(f)}\right|^{2}=2 \cdot(1+\cos (2 \pi f \tau))
$$

The presence of similar-strength signals from two transmitters in a network causes a significant loss of margin. From Equation (23), we can deduce that the resulting channel can have deep "notches" corresponding to the frequency value of $1 / 2 \tau$. The channel characteristics of 2x1 Alamouti MISO called equivalent channel can be expressed by the following equation:

$$
\left(H_{1}{ }^{*}(m, l, p+1) \cdot H_{1(m, l, p)}+H_{2(m, l, p)} \cdot H_{2}{ }^{*}{ }_{(m, l, p+1)}\right)
$$

From these SFN and Alamouti MISO channel equation models, we have simulated the path gain of the different reception case studies. More precisely, Figure 3 gives the path gain simulation results for pure SFN and SFN-2x1 Alamouti MISO transmission from the reception based on two, three, and four transmitters. These results show clearly that in the transmission based on two antennas, there are the deep "notches" for an SFN transmission, which leads transmission cuts. These results demonstrate that an Alamouti MISO transmission allows to obtain better performance than an SFN transmission for two antennas. Thus, we can deduce that the diversity transmission based on two antennas eliminates the notches produced in an SFN network and improves the diversity gain. To justify the reliability of our MISO simulation and verify the functionality of the platform with the considered Alamouti MISO configuration, the reference used to validate our simulation results is based on the comparison of the BER results of the MISO two antennas transmission and the Gaussian channel transmission. Indeed, theoretically the Fourier transform of the Gaussian channel is almost the same that of the MISO two antennas transmission, which eliminates the notches that exist in pure SFN 0-dB Echo transmission. In the case of three transmitters, the simulation results show that the performance of the path gain of the SFN-2x1 Alamouti MISO transmission is still better than that of an SFN transmission although the "notches" for both transmission cases still remain though only the deep "notches" for the SFN transmission. Thereby, we can deduce that the diversity reduces the notches compared to SFN network based on three antennas. In the case of four antennas, both transmissions mode have a bad path gain and are characterized by the deep "notches". Consequently, for four transmitters, the impact of diversity compared to normal transmission (pure SFN) is negligible. From these different case studies, we can note that the efficiency of the SFN-2x1 Alamouti MISO transmission decreases as the number of antennas at the transmission side increases.

Figures 4, 5, 6, and 7 compare the BER performance between the SFN with and without 2x1 Alamouti MISO. These simulation results use the configuration parameters as specified in Table 1 and for different types of

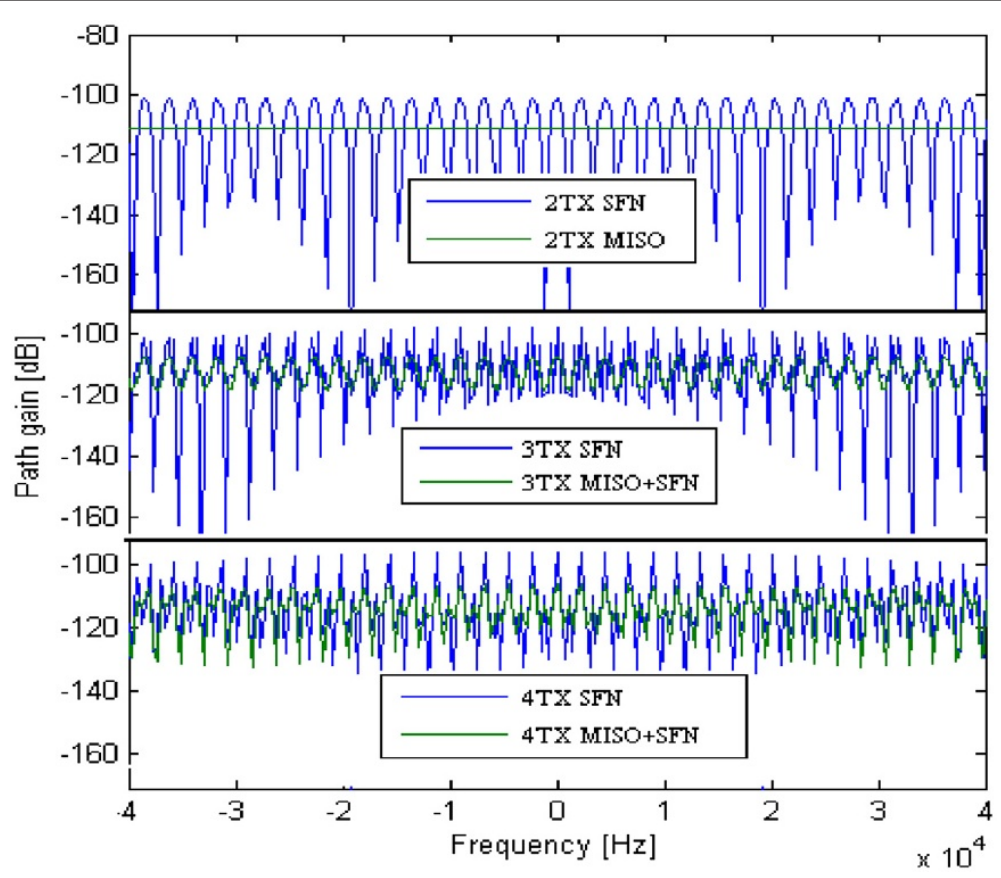

Figure 3 Comparison of the path gain between a SFN with and without Alamouti MISO transmissions for two, three, and four antennas. 


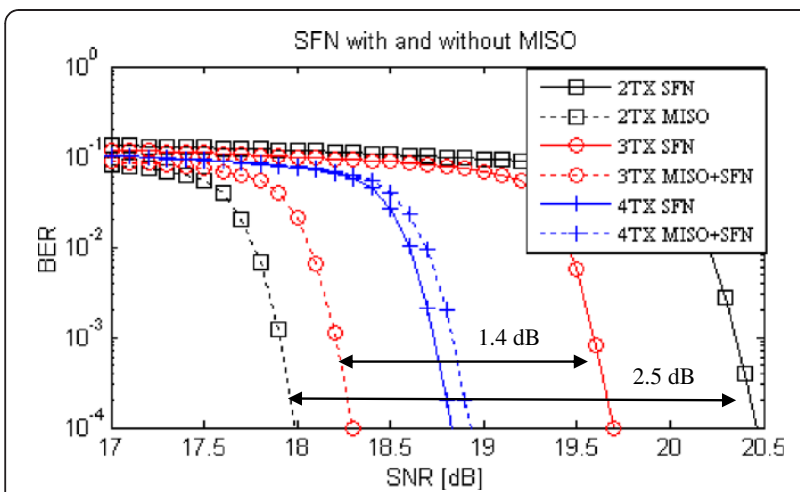

Figure 4 BER performance results for the configuration 256 QAM, 2/3, 32 k, 0-dB ECHO, PP4.

modulations (QPSK, 16 QAM, 64 QAM, and 256 QAM). Indeed, the QEF (quasi-error free) condition of the proposed DVB-T2 configuration is achieved when BER is equal to $10^{-4}$ with a LDPC decoding, which corresponds to a BER of approximately $10^{-7}$ after a $\mathrm{BCH}$ decoding [4]. From these results and in the case of two or three antennas, we observe that whatever the modulation types, an Alamouti MISO transmission (two antennas) or an SFN2x1 Alamouti MISO transmission (three antennas) are more efficient. In an SFN transmission for two or three antennas, the presence of similar-strength signals from the transmitters in a network causes a significant loss of margin because the resulting channel can have deep "notches". These notches are removed by the two antennas (due to the diversity transmission) and their effect are reduced for three antennas with a diversity $2 \times 1$ Alamouti MISO transmission (see Figure 3). Thus, the path gain for an Alamouti MISO transmission is better than the path gain of a pure SFN transmission. However, in the cases of four antennas, we have observed that the BER measurement for an SFN transmission has almost the same performance as an SFN with Alamouti MISO transmission. More precisely, there is no impact of the diversity transmission compared to

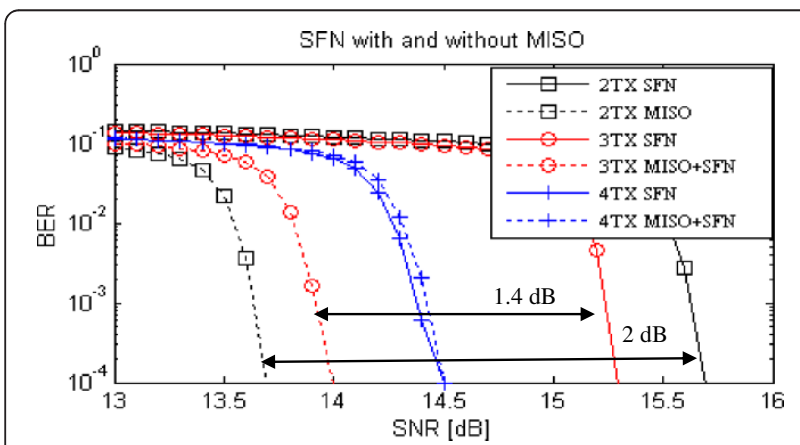

Figure 5 BER performance results for the configuration 64 QAM, 2/3, 32 k, 0-dB ECHO, PP4.

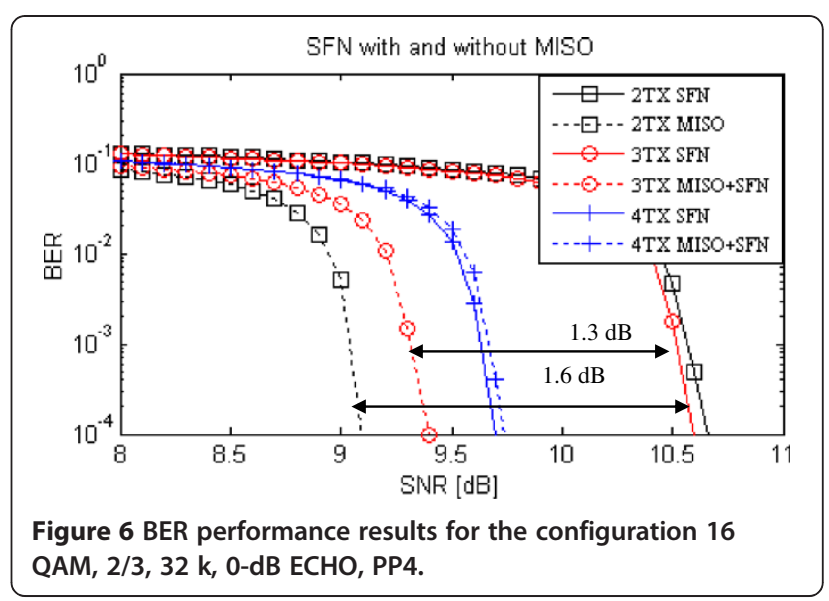

normal transmission for this case studied. This performance is due to the path gain for an SFN-2x1 Alamouti MISO, which is not better than in a classical SFN transmission (see Figure 3). In addition to the similarity in the path gain, the matched filter receiver no longer provides the optimum solution because the matrix $\mathbf{H}$ is no longer orthogonal. Finally, these simulations results show also that in the case of three antennas the gain provided by Alamouti MISO is about $1.4 \mathrm{~dB}$ for all types of modulation. In the case of two antennas, the gain is increased with an increasing constellation size. Thus, Alamouti MISO improves the gain of 1.2, 1.6, 2, and $2.5 \mathrm{dBs}$ for QPSK, 16 QAM, 64 QAM, and 256 QAM modulations, respectively. Therefore, we can deduce that there is no gain in the case of an SFN2x1 Alamouti MISO transmission based on four antennas. For four antennas, there is no gain with an SFN-2x1 MISO Alamouti compared to normal SFN broadcast network. The main explanation is due to the attenuation of two SFN networks caused by the notches of each one. Indeed, these notches are not corrected unlike in the case of two and three antennas where the notches are corrected by the third diversity antenna. As constellation order gets higher, bits

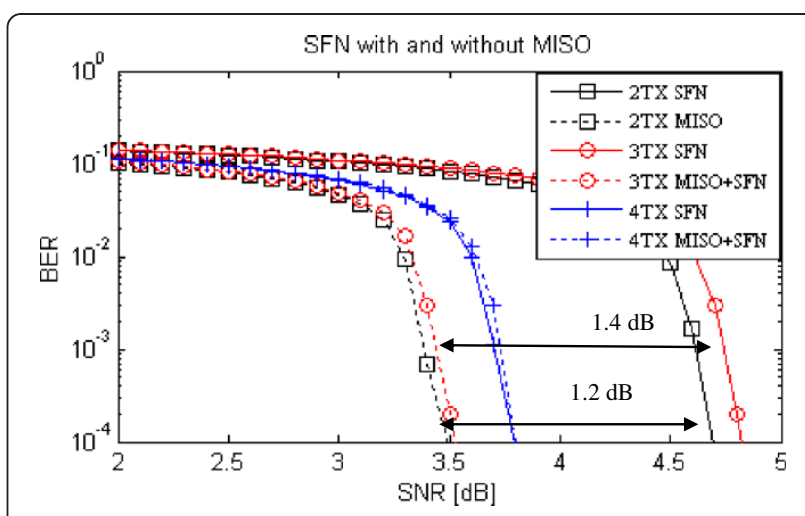

Figure 7 BER performance results for the configuration QPSK, 2/3, 32 k, 0-dB ECHO, PP4. 
per constellation are higher and the distance between the constellations is closer, which makes it more sensitive for the channel fading and noise. Consequently, the reception performance is better as constellation order gets lower at the expense of the less data rate for pure SFN network or SFN-2x1 Alamouti MISO. Compared to pure SFN network, the better gain of SFN-2x1 Alamouti MISO obtained with a higher constellation order is because for a higher constellation order the received performance is more sensitive to the channel fading and noise. Therefore, for the same channel fading, the 2x1 Alamouti MISO diversity corrects more errors compared to lower orders and achieves a better diversity gain. Consequently, these results allow the broadcasters to define an efficient network combination (classical SFN or combined SFN with Alamouti MISO networks) before planning their network forms.

Possible extensions to mobile scenarios are also envisioned for the DVB-T2 [4]. However, actually there are not any performance evaluations of the TU6 in an SFN network with distributed $2 \times 1$ Alamouti MISO for DVB-T2. For example, in mobility conditions the Inter Carrier Interferencedue to Doppler spreading can produce a different impact on the BER performance. Therefore, it is interesting to study the BER performance for different case studies. Consequently, we have also studied and evaluated the performance of a classical SFN network compared to SFN with distributed Alamouti MISO coding over Doppler mobile channel.

\section{Model and evaluation performance of SFN TU6 and MISO TU6}

TU6 channel profile reproduces the terrestrial propagation in an urban area. It has been defined by COST 207 as a typical urban (TU6) profile and is made of six paths having a wide dispersion in delay and relatively strong power. We have measured the performance of DVB-T2 for two transmitters/one receiver system communication in a TU6, SFN and Alamouti MISO channel models on the one hand and combined TU6-MISO, TU6-SFN, and on the other hand. We have considered the mobility (TU6 model) for Doppler frequency $80 \mathrm{~Hz}$ for the DVB-T2. We have supposed that the receiver receives the same power from the two transmit antennas.

In our case study, for modeling an SFN network over TU6 channel, we have considered a classical SFN network of two transmit antennas for a delay of $0.9 \Delta$ with independent TU6 channel on each link [4]. We considered two distributed transmit antennas using Alamouti coding and a single receive antenna with independent TU6 channel on each link for model an Alamouti MISO transmission over TU6 channel.

We describe the BER performance as a function of $C / N$ obtained for DVB-T2 simulations. The parameters used for these simulations are similar to those for Table 1 except for the Mode, Guard Interval, and the Scattered pilot pattern, which are $8 \mathrm{~K}, 1 / 8$, and PP2, respectively. The examined constellation is QPSK and the code rate is $2 / 3$. The QEFcondition is assumed to be achieved when BER is equal to $10^{-7}$ after LDPC decoding and $10^{-11}$ after a $\mathrm{BCH}$ decoder. The Doppler frequency used is equivalent to $118 \mathrm{~km} / \mathrm{h}$ for a carrier frequency of $730 \mathrm{MHz}$ and a data rate corresponding to $7.8 \mathrm{Mbit} / \mathrm{s}$. Therefore, we have adopted a small bit rate allowing robust receivers.

Figure 8 shows the numerical results of these simulations. Comparing the SFN and the SFN with Alamouti MISO, we can deduce that an SFN-2x1 Alamouti MISO transmission yields better performance than classical SFN transmission. This result explains the gain of diversity compared to normal transmission. Indeed, the simulation obtained results show a degradation of the performance of BER using TU6 model. This degradation is less if the TU6 model is used in the classical SFN network. This degradation can be solved by using TU6 model with Alamouti MISO decoding. The obtained results show clearly that the BER performance using TU6 model with MISO decoding is better than that of a classical SFN network for two transmit antennas. Thus, one can minimize the degradation of TU6 for DVB-T2.

\section{Performance evaluation with unbalanced received power}

To evaluate the unbalance received power signals at the receiver, which represent the attenuation of some transmitters compared to others, we have simulated different values of attenuated echo for SFN and SFN-2x1 Alamouti MISO transmissions. We have studied the performance evaluations of SNR over different levels of received power. Thus, we have considered three cases for this simulation. The first case represents the received power from two antennas transmitting two signals corresponding at one direct and one variable power level echo of $0.9 \Delta$. Table 2 presents the

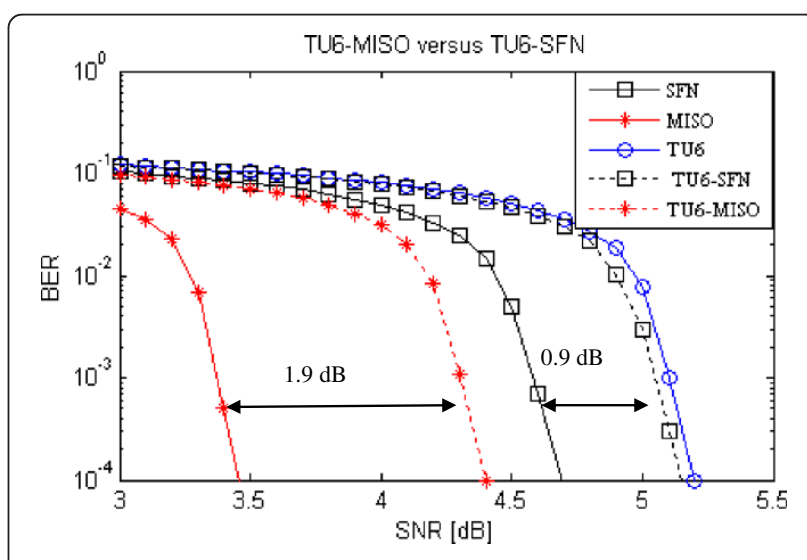

Figure 8 BER as a function of SNR for TU6 channel model with or without SFN and MISO. 
Table 2 Unbalanced received power level results for two, three antennas (in $\mathrm{dB}$ )

\begin{tabular}{|c|c|c|c|c|c|c|}
\hline Direct signal & Echo $0.18 \Delta$ signal & Echo $0.9 \Delta$ signal & SNR for SFN 2 & SNR for MISO-SFN 2 & SNR for SFN 3 & SNR for MISO-SFN 3 \\
\hline 0 & 0 & 0 & 20.4 & 17.9 & 19.6 & 18.3 \\
\hline 0 & 0 & -2 & / & / & 19.7 & 18.3 \\
\hline 0 & 0 & -4 & / & / & 19.9 & 18.2 \\
\hline 0 & 0 & -8 & / & / & 20.5 & 18.1 \\
\hline 0 & -1 & -2 & / & / & 19.7 & 18.2 \\
\hline 0 & -1 & -4 & / & / & 19.9 & 18.2 \\
\hline 0 & -2 & -2 & 19.9 & 17.9 & 19.7 & 18.2 \\
\hline 0 & -2 & -3 & / & / & 19.8 & 18.2 \\
\hline 0 & -2 & -4 & / & / & 19.9 & 18.2 \\
\hline 0 & -3 & -5 & / & / & 20.0 & 18.1 \\
\hline 0 & -4 & -4 & 19.3 & 17.9 & 19.9 & 18.2 \\
\hline 0 & -5 & / & 19 & 17.9 & / & / \\
\hline 0 & -8 & 0 & 18.5 & 17.9 & 20.0 & 18.3 \\
\hline
\end{tabular}

SNR results for the SFN and Alamouti MISO networks for two and three antennas.

We have deduced that the SNR value for two antennas decreases when the attenuation level of the echo signal increases. Then, it reaches a Ricean channel performance value when the attenuated value increases further. However, for the Alamouti MISO transmission technique-based network, the SNR value remains stable. This can be explained by the Fourier transform of the channel of SFN-2x1 Alamouti MISO network represented in Figure 3 where it can be seen that the 2x1 Alamouti MISO equivalent channel is constant. However, for an SFN the notches will be recovered and reduced by the attenuated power level of the echo. Thus, this will give better SNR performance.

The second case study represents the received signals from three antennas transmitting three signals corresponding at one direct and two variable power level echo of $0.18 \Delta$ and $0.9 \Delta$, respectively. The direct and echo $0.9 \Delta$ signals transmit the same symbols (mode $A$ ) while the echo $0.18 \Delta$ signal transmits conjugate symbols (mode $B$ ). Again, we can deduce that in the case of an SFN-2x1 Alamouti MISO, the SNR is almost constant when the echo $0.18 \Delta$ is attenuated. These results can be explained as if we have an Alamouti MISO of two transmitters. The first one is the combined signal pair of direct and echo $0.9 \Delta$ while the second is the echo $0.18 \Delta$, which becomes similar to the first case. However, if the second echo $(0.9 \Delta)$ is attenuated the SNR values tend towards those of an $2 \times 1$ Alamouti MISO technique network based on two antennas.

The third studied case is concerned with the received power level from four antennas transmitting four signals corresponding to one direct signal and three echo signals of $0.18 \Delta, 0.72 \Delta$, and $0.9 \Delta$, respectively. The direct and echo $0.9 \Delta$ signals transmit the same symbols while the echo signals of $0.72 \Delta$ and $0.18 \Delta$ transmit the conjugate symbols.
Table 3 lists the SNR evaluations obtained. These results show that if we attenuate one of the four antennas, an SFN-2x1 Alamouti MISO network tends to work as an SFN-2x1 Alamouti MISO based on three antennas only (second case studied).

More precisely, this case has the same evaluation result as an SFN-2x1 Alamouti MISO network based on three antennas. If we attenuate two antennas (antenna 2 or 3 and antenna 4 at the same time) corresponding to two pairs of antennas modeling two SFN networks (the first one is the combined signal pair of direct and echo $0.9 \Delta$ while the second is the combined of echo $0.18 \Delta$ and $0.72 \Delta$ ), then these two networks form an SFN-2x1 Alamouti MISO. The SNR value depends on the attenuated echo of each pair of SFN. In addition, if we attenuate two antennas transmitting the same symbols (for

Table 3 Unbalanced received power level results for four antennas (in $\mathrm{dB}$ )

\begin{tabular}{llllll}
\hline $\begin{array}{l}\text { Direct } \\
\text { signal }\end{array}$ & $\begin{array}{l}\text { Echo } \mathbf{0 . 1 8} \\
\boldsymbol{\Delta} \text { signal }\end{array}$ & $\begin{array}{l}\text { Echo } \mathbf{0 . 7 2} \\
\boldsymbol{\Delta} \text { signal }\end{array}$ & $\begin{array}{l}\text { Echo 0.9 } \\
\boldsymbol{\Delta} \text { signal }\end{array}$ & $\begin{array}{l}\text { SNR } \\
\text { for SFN }\end{array}$ & $\begin{array}{l}\text { SNR for } \\
\text { MISO-SFN }\end{array}$ \\
\hline 0 & 0 & 0 & 0 & 18.8 & 18.9 \\
0 & 0 & 0 & -2 & 18.9 & 18.8 \\
0 & 0 & 0 & -5 & 19.2 & 18.6 \\
0 & 0 & 0 & -8 & 19.4 & 18.5 \\
0 & 0 & -5 & 0 & 19.1 & 18.6 \\
0 & 0 & -2 & -5 & 19.2 & 18.6 \\
0 & 0 & -10 & 0 & 19.3 & 18.4 \\
0 & 0 & -8 & -8 & 19.2 & 18.2 \\
0 & -2 & -5 & -5 & 19.1 & 18.6 \\
0 & -5 & -5 & -5 & 19.1 & 18.6 \\
0 & -10 & -10 & 0 & 19.5 & 18.9 \\
\hline
\end{tabular}


instance antenna 2 and 3 or antennas 1 and 4 at the same time), the resulting SNR tends to be stable at 18.9 $\mathrm{dB}$ and behaves as an SFN-2x1 Alamouti MISO network with two antennas. Indeed, this case represents two pair of SFN networks that undergoes a diversity transmission signals. One of these two SFN networks is attenuated and refers to the case of an attenuated echo for MISO based on two transmitters. However, if three echo signals are attenuated, the network behavior depends on the attenuation levels while the value of SNR depends on the attenuated echoes.

\section{Performance evaluation with rotated constellations}

The rotated constellations, which provide a form of modulation diversity, are useful to assist in the reception of higher-code-rate signals in demanding transmission channels $[26,27]$. The technique of rotated constellations proposed by the DVB-T2 standard consists of applying a rotation to the QAM constellation followed by a component-axes interleaving. The DVB-T2 is not limited by the use of the obtained full rank transmission matrices. Indeed, it is the interleaving with constellation rotation which improves the diversity performance. The principles use of rotated constellation is as follows [28]. In a nonrotated constellation, the receiver needs both in phase (I) and quadrature $(\mathrm{Q})$ components of one constellation point to identify what information was transmitted, because the estimation of I does not give information about Q component. Besides, both components suffer the same fading when the signal is propagated over the channel. In the case of a rotated constellation, a certain rotation angle is applied in the complex plane to a classical signal constellation, such that each component, I or Q, has enough information by its own to estimate what transmitted symbol was. After the rotation, an interleaving process is performed only over the Q components [28]. This is done in order to transmit separately the I and Q of a constellation point in different carriers and even in different time slots. Thus, if one of the components is destroyed or affected by a deep selective fading of the channel, the other component can be used to recover the information. In addition, due to this interleaving process, the in-phase and quadrature components of a transmitted symbol are affected by independent fading. The result of this technique is to increase the robustness of the receiver in propagation scenarios with deep fades and/or erasure events. Therefore, after rotating, the projections of the constellation points on the I and Q channels carry the information regarding the $m$ mapped bits. For a 16-QAM, instead of $2^{m / 2}=4$ projections on each axis, the constellation now has $2^{m}=16$ projections as seen in Figure 9 [4].

Thereby, the insertion of the interleaving between I and $Q$ leads to the same information being sent twice over the channel in different cells, as if an inner

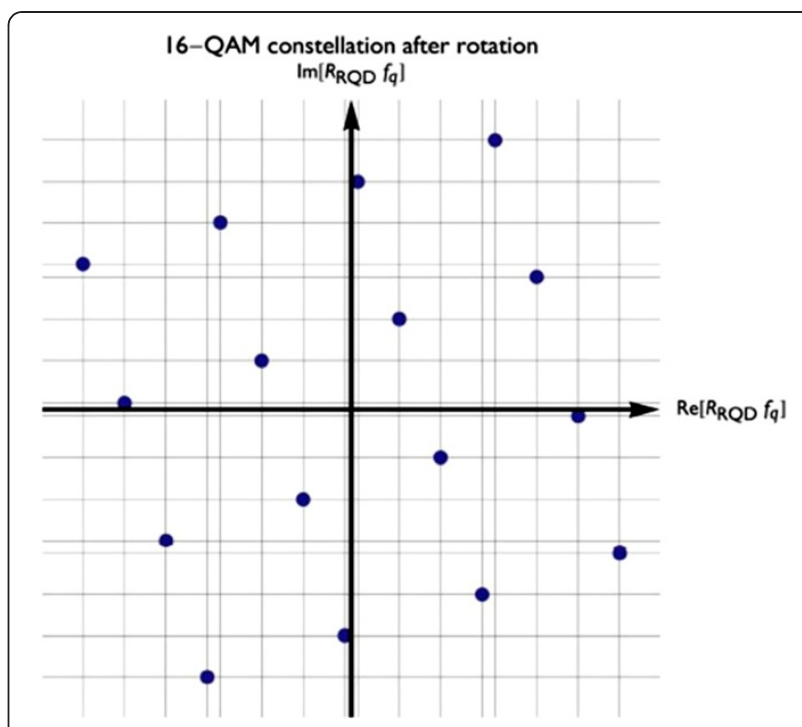

Figure 9 16-QAM constellation after rotation.

repetition code was used. The resulting "virtual" constellation after rotation and cyclic delay in the case of a 16QAM is shown in Figure 10 [4].

It is equivalent to sending a higher-order irregular QAM while having the spectral efficiency of the original QAM. This leads to additional diversity that improves the error-correcting performance when severely faded channels are encountered [4]. The component-axes (or I/Q) interleaving is performed by using a cyclic delay before cellinterleavers and time-interleavers. The optimum choice of the angle is dependent on modulation order, channel type,

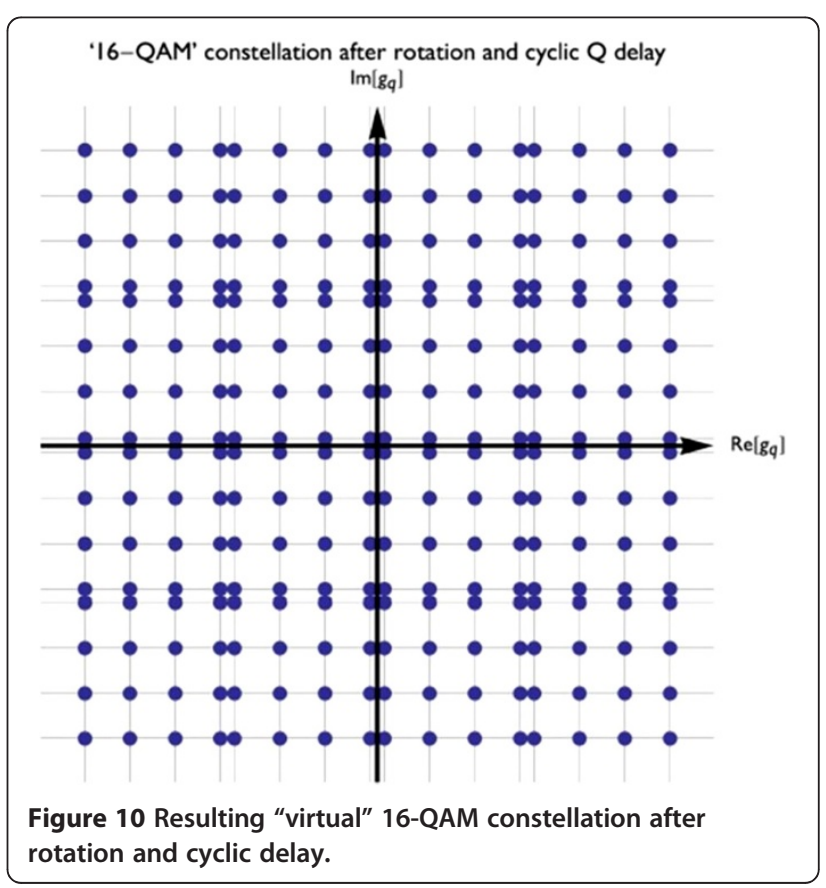


and mapping type. For each modulation order (constellation size), a single corresponding rotation has been chosen. In this section, we study the performance of rotated constellations for the previous different configurations used. More precisely, we will study the performance of rotated constellation for SFN-2x1 Alamouti MISO network with the configuration specified in Table 1 and TU6 channel with the configuration specified in Section 3. The results are shown in Table 4. This table gives the results of the SNR performance for different types of networks (SFN, SFN-2x1 Alamouti MISO) with and without rotated constellations over different numbers of antennas, and different types of constellations. Therefore, for instance in this table, the 256 QAM-2 means that the number of antennas is two and the constellation is 256 QAM. Note that the gain obtained by using a rotated constellation also depends on the receiver implementation. The worst case theoretically for the simplest implementation is supposed to obtain the same performance whether the rotated constellation can be used or not. These results show that for diversity transmissions, the values of SNR are almost stable with and without rotated constellations for all cases except for the TU6-MISO case where a deviation of $0.4 \mathrm{~dB}$ was obtained. For the pure SFN network case, the values of SNR depend on the type of modulation. For the 256 QAM modulations, the values are almost stable for rotated constellations in active and no-active modes. However, for others modulations (64 QAM, 16 QAM, QPSK) the deviation increases when bits per constellation decreases. For QPSK, the highest deviation was obtained.

\section{Conclusion}

This article addresses a new model including the simulation results and gives a detailed analysis of the performance of the Alamouti code in an SFN for DVB-T2 for two, three, and four transmitters, different types of coding, and modulations. We have studied and evaluated the expected performance for the transmission-based Alamouti MISO technique, which is an option in the DVB-T2 standard. Our proposed model has been implemented in the CSPDVB-T2 platform. To achieve this, we have proposed a new model DVB-T2 configuration to simulate an SFN network transmission with Alamouti MISO in order to improve the quality of the received signals. The simulation results obtained show clearly that in the case of two, three, or four transmission antennas, the performance of SFN (0-dB Echo profile) with Distributed Alamouti MISO is only better than that of a classical SFN for two and three antennas. However, in the case of four antennas, the results obtained show clearly that a classical SFN transmission has almost the same performance as an SFN with Alamouti MISO. These simulation results show also that in the case of three antennas the gain provided by Alamouti MISO is bigger than $1.5 \mathrm{~dB}$ for all types of modulation. In the case of two antennas, the gain is increased with bits per constellation. Consequently, Alamouti MISO improves the gain by 1.2, 1.6, 2, and $2.5 \mathrm{~dB}$ for QPSK, 16 QAM, 64 QAM, and 256 QAM modulations, respectively. Finally, we deduce that there is no gain only for the case of an SFN with Alamouti MISO transmission based on four antennas.

We conclude that the reception performance obtained with an SFN-2x1 Alamouti MISO is better than a pure SFN network when constellation order gets lower. Indeed, for the same channel fading, the $2 \times 1$ Alamouti MISO diversity corrects more errors when compared to lower orders and, then, achieves a better diversity gain.

We have also shown that the unbalanced received power has no effect for Alamouti MISO transmission (MISO two antennas). However, some effects appear for the SFN and SFN-2x1 Alamouti MISO networks. More precisely, we have demonstrated that SFN or SFN-2x1 Alamouti MISO networks based on four antennas behave like a threeantenna SFN or SFN-2x1 Alamouti MISO networks, or three antennas SFN or SFN-2x1 Alamouti MISO networks operate like a two-antenna SFN or SFN-2x1 Alamouti MISO networks depending on the echo attenuation. In the case of the rotated constellation study, only the SFN network undergoes the effects when the deviation between the rotated constellations is active depending on the modulation type. Thus, we show that the expected results

Table 4 Rotated constellation effects in term of SNR contributions for the used MISO-SFN configuration, TU6-MISO, and TU6-SFN configurations (in $\mathrm{dB}$ )

\begin{tabular}{|c|c|c|c|c|c|c|c|c|c|c|}
\hline $\begin{array}{l}\text { Antennas } \\
\text { number }\end{array}$ & $\begin{array}{l}\text { SFN with } \\
\text { Rot.cons. }\end{array}$ & $\begin{array}{l}\text { SFN } \\
\text { without } \\
\text { Rot.cons. }\end{array}$ & $\begin{array}{l}\text { MISO SFN } \\
\text { with Rot.cons. }\end{array}$ & $\begin{array}{l}\text { MISO SFN } \\
\text { without } \\
\text { Rot.cons. }\end{array}$ & $\begin{array}{l}\text { TU6 with } \\
\text { Rot.cons. }\end{array}$ & $\begin{array}{l}\text { TU6 without } \\
\text { Rot.cons. }\end{array}$ & $\begin{array}{l}\text { TU6 SFN } \\
\text { with Rot.cons. }\end{array}$ & $\begin{array}{l}\text { TU6 SFN } \\
\text { without } \\
\text { Rot.cons. }\end{array}$ & $\begin{array}{l}\text { TU6 MISO } \\
\text { with Rot.cons. }\end{array}$ & $\begin{array}{l}\text { TU6 MISO } \\
\text { without } \\
\text { Rot.cons. }\end{array}$ \\
\hline QPSK-2 & 4.6 & 5.9 & 3.4 & 3.4 & 5.2 & 6.4 & 5.1 & 6.3 & 4.4 & 4.8 \\
\hline 16 QAM-2 & 10.6 & 11.4 & 9.1 & 9.1 & / & / & / & / & / & / \\
\hline 64 QAM-2 & 15.6 & 16 & 13.6 & 13.6 & / & / & / & / & / & / \\
\hline 256 QAM-2 & 20.4 & 20.5 & 18 & 17.9 & / & / & / & / & / & / \\
\hline 256 QAM-3 & 19.6 & 19.7 & 18.2 & 18.2 & / & / & / & / & / & / \\
\hline 256 QAM-4 & 18.8 & 18.8 & 18.9 & 18.9 & / & / & / & / & / & / \\
\hline
\end{tabular}

Rot. Cons.,rotated constellation. 
demonstrate that broadcasters who consider that Alamouti MISO always give a performance better than SFN are not always correct. These results are very important for the broadcaster who will set the operational management planning and the network deployment. Indeed, depending on whether an SFN-2x1 Alamouti MISO transmission gives better performance than a classical SFN transmission, or not, the broadcaster defines the appropriate transmission network.

The results obtained also suggest that the BER performance using TU6 model with Alamouti MISO decoding performs better than when using a classical SFN network with two transmitting antennas. This allows us to minimize the degradations of TU6 for DVB-T2. The results converge as expected with the comparison between a classical SFN and an SFN-2x1 Alamouti MISO. This trend will need to be investigated in more detail as future work. Other future work will be to study and evaluate the BER performance for the same channel models when using other Doppler frequencies in a network of more than two antennas.

\section{Competing interests}

The authors declare that they have no competing interests.

\section{Author details}

'Interfaces, Sensors and Microelectronics laboratory (LICM), University of Lorraine at Metz, 7 rue Marconi, 57070, Metz, France. ${ }^{2}$ Télédiffusion de France (TDF), 1 rue Marconi, 57070, Metz, France.

Received: 20 June 2011 Accepted: 4 February 2013

Published: 19 March 2013

\section{References}

1. ETSI EN 300744 V1.6.1, Digital Video Broadcasting (DVB), Framing structure, channel coding and modulation for digital terrestrial television, European Standard Telecommunications series, European Broadcasting Union (ETSI, 2009)

2. ETSI TR 101190 V1.3.1, Digital Video Broadcasting (DVB), Implementation guidelines for DVB terrestrial services; Transmission aspects, European Standard Telecommunications series, European Broadcasting Union (ETSI, 2008)

3. ETSI TS 101191 V1.4.1, Digital Video Broadcasting (DVB), DVB mega-frame for Single Frequency Network (SFN) synchronization, European Standard Telecommunications series, European Broadcasting Union (ETSI, 2004)

4. ETSI TR $102831 \mathrm{~V}<0.8 .57>$ Digital Video Broadcasting (DVB), Implementation guidelines for a second generation digital terrestrial television broadcasting system (DVB-T2, European Standard Telecommunications series, European Broadcasting Union) (ETSI, 2008)

5. H Bölcskei, AJ Paulraj, Space-frequency coded broadband OFDM systems. inProceedings of the IEEE Wireless Communication Network Conference (WCNC) 1, 16 (2000)

6. J Yang, K Cheun, Low complexity implementation of Alamouti space-time coded OFDM transmitters. IEEE Commun Lett 8(4), 229-231 (2004)

7. A Hutter, S Mekrazi, BN Getu, F Platbrood, Alamouti-based space-frequency coding for OFDM. Int. J. Wirel. Personal Commun. 35(1-2), 173-185 (2005)

8. W Su, Z Safar, KJR Liu, Full-rate full-diversity space-frequency codes with optimum coding advantage. IEEE Trans Inf Theory 51(1), 229-249 (2005)

9. K Lee, D Williams, A space-frequency transmitter diversity technique for OFDM systems. inProceedings of IEEE GLOBECOM 3, 14731477 (2000)

10. ETSI EN 302755 V1.1.1, Digital Video Broadcasting (DVB), Frame structure channel coding and modulation for a second generation digital terrestrial television broadcasting system (DVB-T2) (ETSI, 2009)

11. SM Alamouti, A simple transmit diversity technique for wireless communications. IEEE J. Sel. Areas Commun. 16(8), 1451-1458 (1998)
12. M Poggioni, L Rugini, P Banelli, DVB-T/H and T-DMB: Physical layer performance comparison in fast mobile channels. IEEE Trans Broadcast 55(4), 719-730 (2009)

13. S Ruiz-Boqué, C Lopez, C Enrique, L Alonso, J Olmos, M Garcia, F Minerva, Performance of DVB-T2 improvements over low Doppler mobile channels, European Cooperation in the field of scientific and technical research, COST 2100 TD(08) 451 (Wroclaw, Poland, 2008)

14. L Kondrad, VKM Vadakital, I Bouazizi, M Gabbouj, Cross layer optimization of DVB-T2 system for mobile services. Hindawi J. Dig. Multimed. Broadcast 2010, Article ID 435405 (2010)

15. A Vigato, S Tomasin, L Vangelista, V Mignone, N Benvenuto, A Morello, Coded decision directed demodulation for second generation digital video broadcasting standard. IEEE Trans Broadcast 55(3), 607-615 (2009)

16. M Anders, Single frequency network in DTV. IEEE Trans Broadcast 51(4), 1432-1436 (2005)

17. B Badic, M Rupp, H Weinrichter, Adaptive channel matched extended Alamouti space-time code exploiting partial feedback. ETRI J 26(5), 443451 (2004)

18. MR McKay, IB Collings, Capacity and performance of MIMO-BICM with zero-forcing receivers. IEEE Trans Commun 53(1), 74-83 (2005)

19. V Tarokh, H Jafarkhani, A Calderbank, Space-time block codes from orthogonal designs. IEEE Trans Inf Theory 45, 1456-1467 (1999)

20. V Tarokh, H Jafarkhani Et, AR Calderbank, Space-time block coding for wireless communications: performance results. IEEE J. Sel. Areas Commun. Inf. Theory 17, 451-460 (1999)

21. V Tarokh, N Seshadri, A Calderbank, Space-time codes for high data rate wireless communication: performance criterion and code construction. IEEE Trans Inf Theory 44, 744-765 (1998)

22. M Tormos, C Tanougast, A Dandache, P Bretillon, P Kasser, Performance evaluation of distributed Tarokh SFBC and Alamouti MISO for SFN DVB-T2 broadcast networks, in inProceedings of the IEEE International Conference on Electronics, Circuits and Systems, IEEE Circuits and Systems Society, 2011, p. 583586

23. DVB NGH, TM-H NGH Study mission report, Standard Telecommunications series, European Broadcasting Union, 2008, p. TM 4026r

24. O Haffenden, DVB-T2: The common simulation platform, Research white paper, British Broadcasting Corporation (2011), p. WHP 196

25. Mathworks, Matlab Software, Version 7.8.0 (R2009a), The Mathworks Inc. (Massachusetts (USA), 2009)

26. C Abdel Nour, C Douillard, Rotated QAM constellation to improve BICM performance for DVB-T2, inProceedings of the International Symposium on Spread Spectrum Techniques and Applications (ISSSTA 2008) (Bologna (Italy), 2008), pp. 354-359. Patent no FR 2927754, 02

27. M Tormos, C Tanougast, P Bretillon, P Kasser, A Dandache, "Experimental performance of mobile DVB-T2 in SFN and distributed MISO Network", in Proceedings of the IEEE International Conference on Telecommunications (ICT 2012) (Jounieh (Labanon), 2012), pp. 1-5

28. D Perez-Calderón, C Oria, J García, P López, V Baena, I Lacadena, Rotated constellations for DVB-T2, in Proceedings of the Design of Circuits and Integrated Systems Conference (DCIS 2009) (Zaragoza (Spain), 2009), pp. $41-45$

doi:10.1186/1687-1499-2013-78

Cite this article as: Tormos et al: Modeling and performance evaluations of Alamouti technique in a single frequency network for DVB-T2.

EURASIP Journal on Wireless Communications and Networking 2013 2013:78.

\section{Submit your manuscript to a SpringerOpen ${ }^{\circ}$ journal and benefit from:}

- Convenient online submission

- Rigorous peer review

- Immediate publication on acceptance

- Open access: articles freely available online

- High visibility within the field

- Retaining the copyright to your article

Submit your next manuscript at $\gg$ springeropen.com 